\title{
Partial pneumoencephalography in following-up pituitary tumours ${ }^{1}$
}

\author{
WILLIAM W. OLMSTED, GABRIEL H. WILSON, \\ ROBERT W. RAND AND JOHN P. GARTLAND \\ From the Departments of Radiological Sciences and Surgery-Neurosurgery, \\ UCLA Center for the Health Sciences, Los Angeles, and the Department of Radiology, \\ St. Mary's Hospital and Medical Center, San Francisco, California, U.S.A.
}

SYNOPSIS The 'limited' pneumoencephalogram has been used with excellent success at UCLA for the continuing follow-up of pituitary tumours. It is most useful in following nonsecretory adenomas since tumour regrowth can occur in the absence of clinical signs and symptoms. Totat. serial pneumoencephalography has not been accepted previously for follow-up of pituitary tumours? since there is a significant morbidity. The 'limited' pneumoencephalogram of the diseased areag drastically reduces the morbidity of the procedure so that the patients are willing to undergo seriatu studies on an outpatient basis.

Recurrence of secretory pituitary tumours after surgery and/or radiation therapy is usually detected by exacerbation of symptoms and increase in particular hormonal levels-for example, growth hormone $(\mathrm{HGH})$, prolactin, corticotrophin (ACTH). Recurrence of nonsecretory pituitary tumours is more difficult to evaluate because these biochemical parameters are not available. Often tumour tissue can extend well beyond the sella turcica without producing clinical disturbance of the visual pathways. In addition, because visual fields may demonstrate persistent abnormality after therapy, they may be an unreliable indicator of tumour recurrence and extension.

Investigators have attempted to assess tumour recurrence by evaluation of serial lateral radiographs of the sella turcica (Lewtas, 1966), by displacement on serial skull radiographs of silver clips attached to the capsule of the tumour during surgery (sellar tumours) (Wilson and Norrell, 1968), and by the use of tantalum powder (gliomas) (Lindgren et al., 1957). Serial pneumoencephalography in the absence of symptoms has not been an accepted mode of follow-up because the usual pneumoencephalo-

${ }^{1}$ Supported in part by Special NINDS Training Grants 1 F11 NS 2627-01 NSRA (W.W.O.) and 1 F11 NS 2428-01 NSRA (J.P.G.). graphic procedure causes significant morbidi⿻日禸 and hospitalization is required for at least hours after the procedure.

Recently at UCLA Center for the Hea $\frac{1}{10} \overrightarrow{\mathrm{P}}$ Sciences 'limited' pneumoencephalograms hay been performed routinely on pituitary tumout patients after surgery and/or radiation therapy응 The 'limited' approach allows evaluation of thes parasellar and suprasellar areas within a fewo weeks of such therapy, thus establishing a base line and demonstrating the extent of tumoup eradication. The patient is then restudied as an $\overrightarrow{\vec{b}}$ outpatient at specified intervals-for example every 12 to 18 months - to determine if there has been recurrence of tumour and also to evaluate further the extent of tumour eradication where radiation therapy has been used. Morbidity is substantially reduced by the "limited technique and hospitalization is thus unneces. sary.

\section{METHODS}

The patient is admitted to the surgery outpatien recovery room at $8 \mathrm{a} . \mathrm{m}$. on the morning of the study having taken nothing by mouth for the previous 12 hours. A brief outpatient workup is performeof and any appropriate laboratory testing done betweeng the hours of 8 and 10 a.m. At 10 a.m. the patient is 


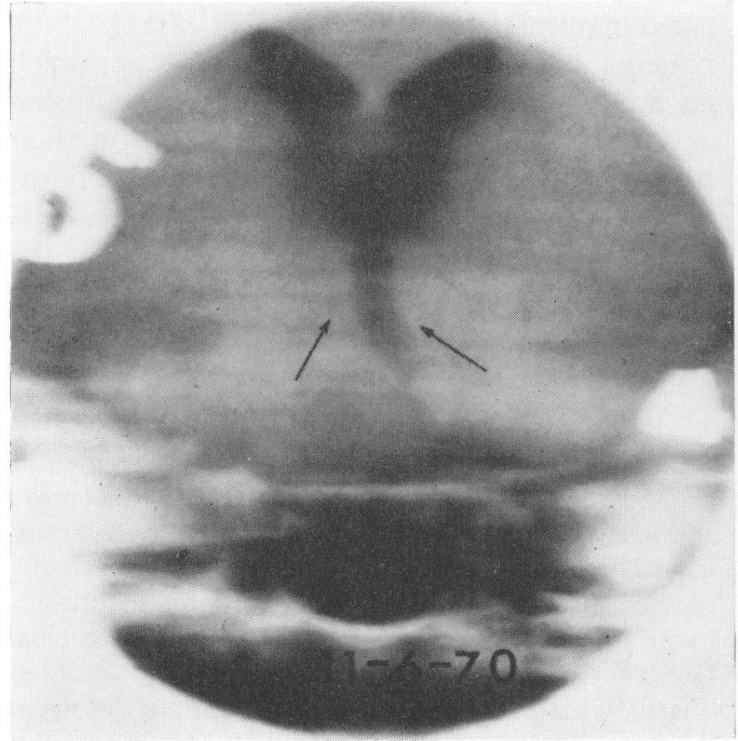

(a)

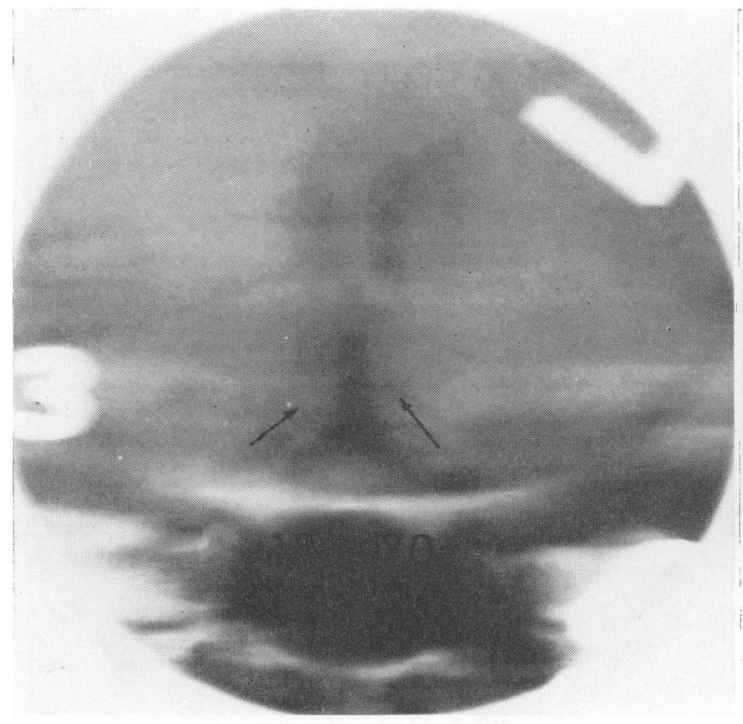

(c)

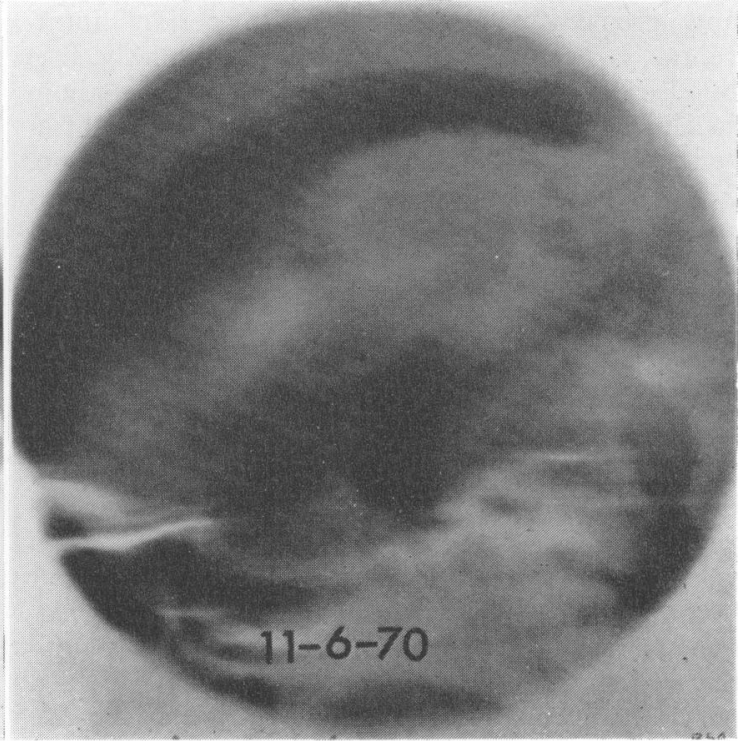

(b)

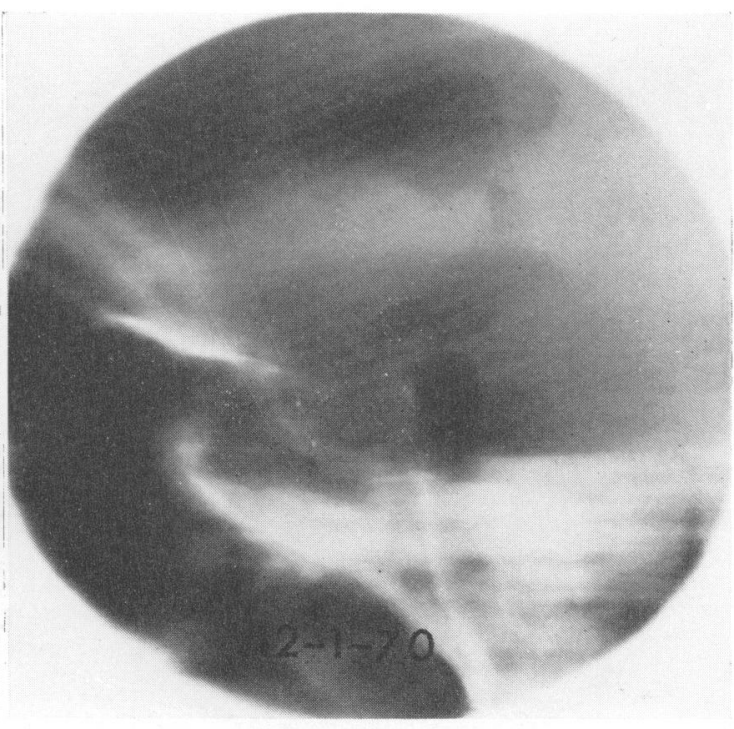

(d)

FIG. 1. Case 1. (a) and (b) Pneumoencephalography before surgery shows a large intrasellar mass with suprasellar extension deviating the third ventricle from right to left. (c) and (d) The post-treatment pneumoencephalogram reveals no suprasellar mass. The third ventricle has returned to the midline and the anterior recesses of the third ventricle are now sharp. 
brought to the Department of Radiological Sciences and premedicated with $2 \mathrm{ml}$ droperidol intravenously and $50 \mathrm{mg}$ ephedrine intramuscularly. One hundred milligrams of hydrocortisone sodium succinate (Solucortef) is generally given intravenously. Lumbar puncture is performed with a no. 22 gauge spinal needle. No cerebrospinal fluid is withdrawn. Sufficient oxygen is injected in flexion to insure adequate visualization of the third ventricle (usually less than $15 \mathrm{ml}$ ). The patient's head is extended and the parasellar cisterns filled with about $10 \mathrm{ml}$ oxygen. Lateral laminograms of the sellar area are obtained in extension. The patient is then placed supine and laminograms are obtained in the anteroposterior and lateral projections. After satisfactory filming the needle is withdrawn and the patient returned to the outpatient recovery room where observation is continued for a period of four hours. The patient is discharged home with analgesic medication and instructed to lie flat in bed until the next morning. An instruction sheet is provided which includes phone numbers of physicians to call in case there are any problems or questions.

\section{RESULTS}

Initially the 'limited' studies were done on an inpatient basis. We have had the opportunity to $z$ use our outpatient protocol in five cases. All the patients examined by this 'limited' approach have felt subjectively that their headache during and after the procedure was less than on their 0 previous encounters. There were no complications of the studies performed and all patients were discharged home on the afternoon of the examination, being virtually asymptomatic at that time.

\section{CASE 1}

S.P., a 24 year old female, presented with amenorrhoea and infertility for six years. During this period she had also complained of weight gain and hirsutism. Three weeks before admission she noticed de- $\overrightarrow{0}$ creased vision in the left eye. Skull films before admission demonstrated an enlarged sella turcica. General physical examination showed marked obesity with no other positive findings. Neurological examination was normal with the exception of a $w_{y}$ dense left homonomous hemianopsia. Carotid arterio ${ }^{2}$ के graphy demonstrated slight elevation of boti proximal anterior cerebral arteries, right greate than the left. A pneumoencephalogram done o⿺

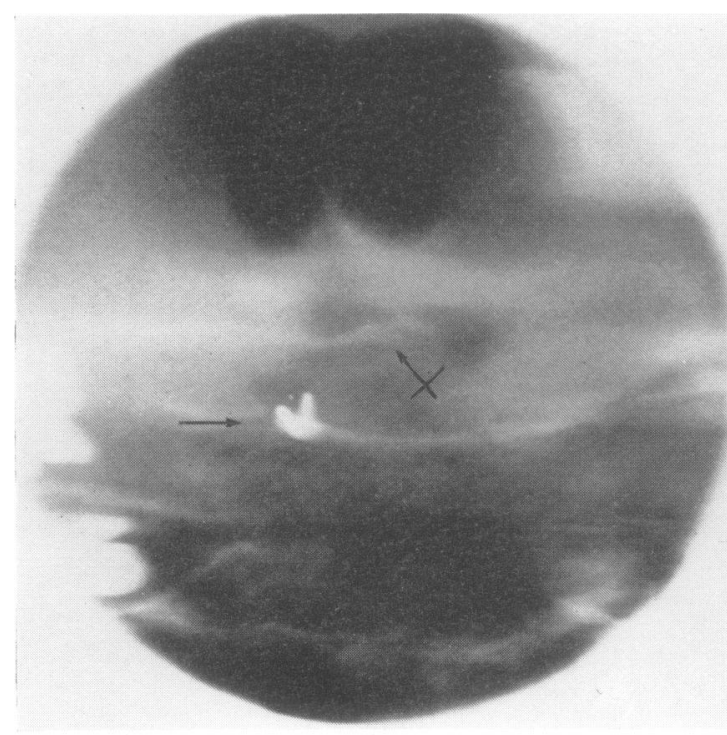

(a)

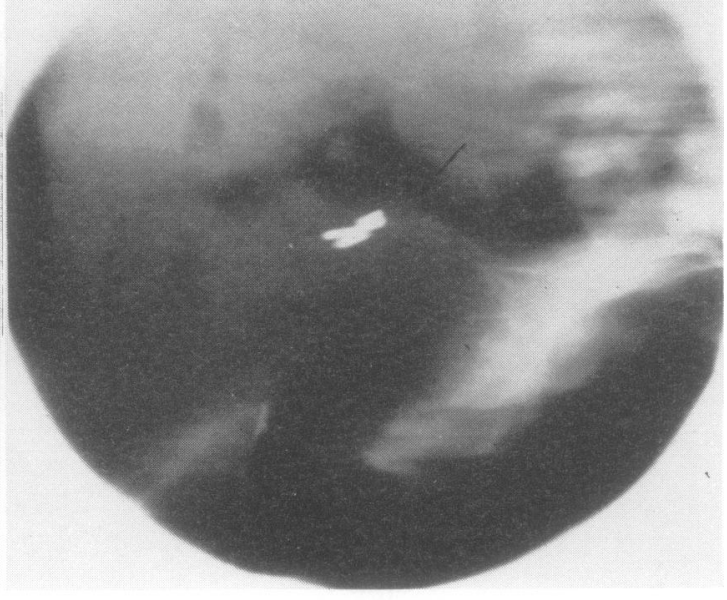

(b)

FIG. 2. Case 2. The plain arrow points to metallic clips left on the tumour capsule at the time of the patient's original surgery. They lie just anterior to the dorsum. The crossed arrow shows indentation of the anterior third ventricle from recurrent tumour. 


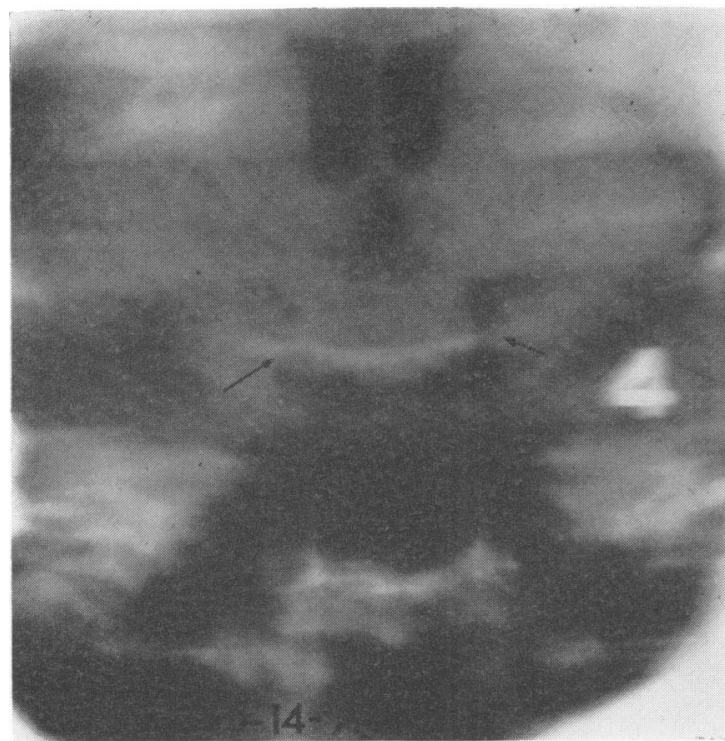

(a)

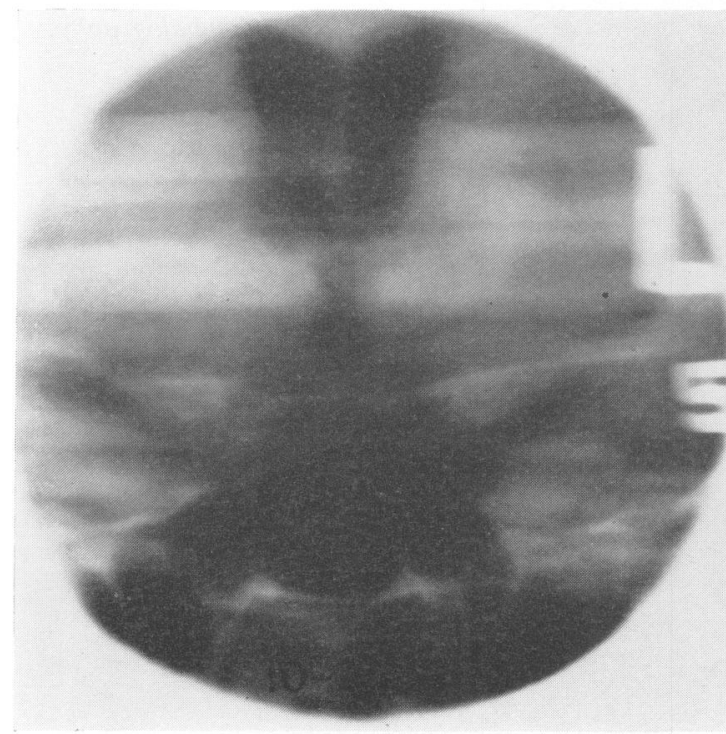

(c)

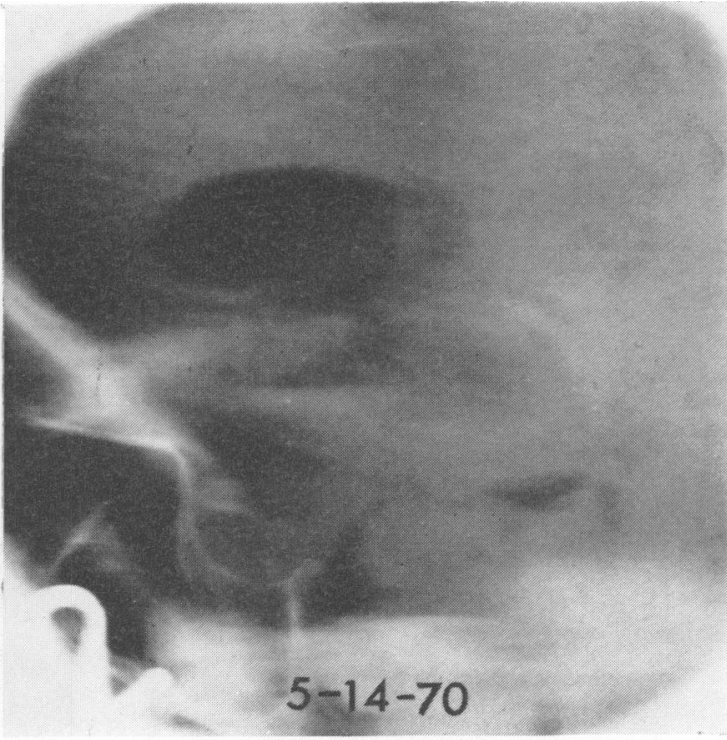

(b)

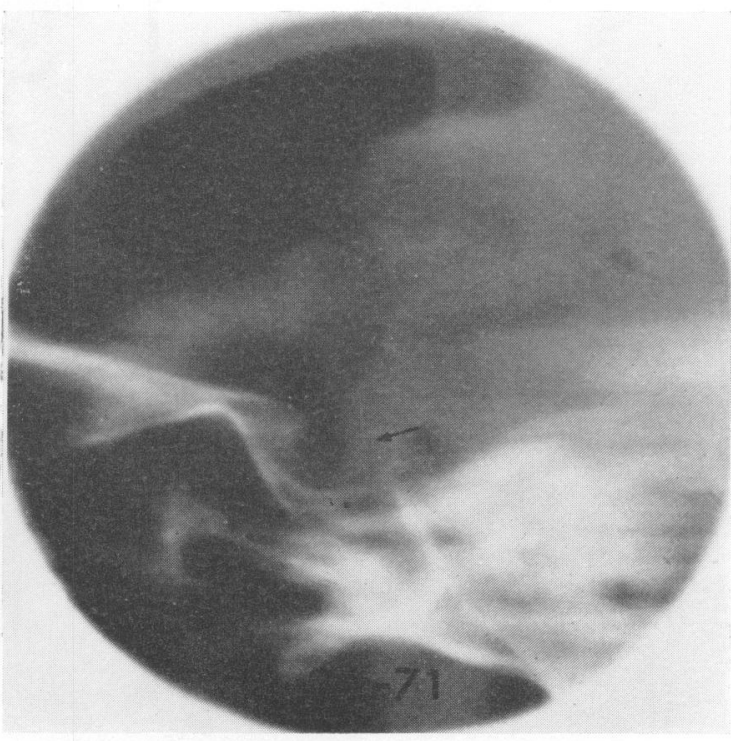

(d)

FIG. 3. Case 3. (a) and (b) The pretreatment pneumoencephalogram reveals splaying of the anterior recesses of the third ventricle from suprasellar extension of intrasellar mass. Arrows indicate the extent of the tumour laterally. The floor of the third ventricle is indented on the AP laminogram. (c) and (d) Post-treatment pneumoencephalography reveals that the anterior recesses of the third ventricle are now in normal position. The arrow indicates oxygen in the sella turcica and thus an 'empty sella'. 


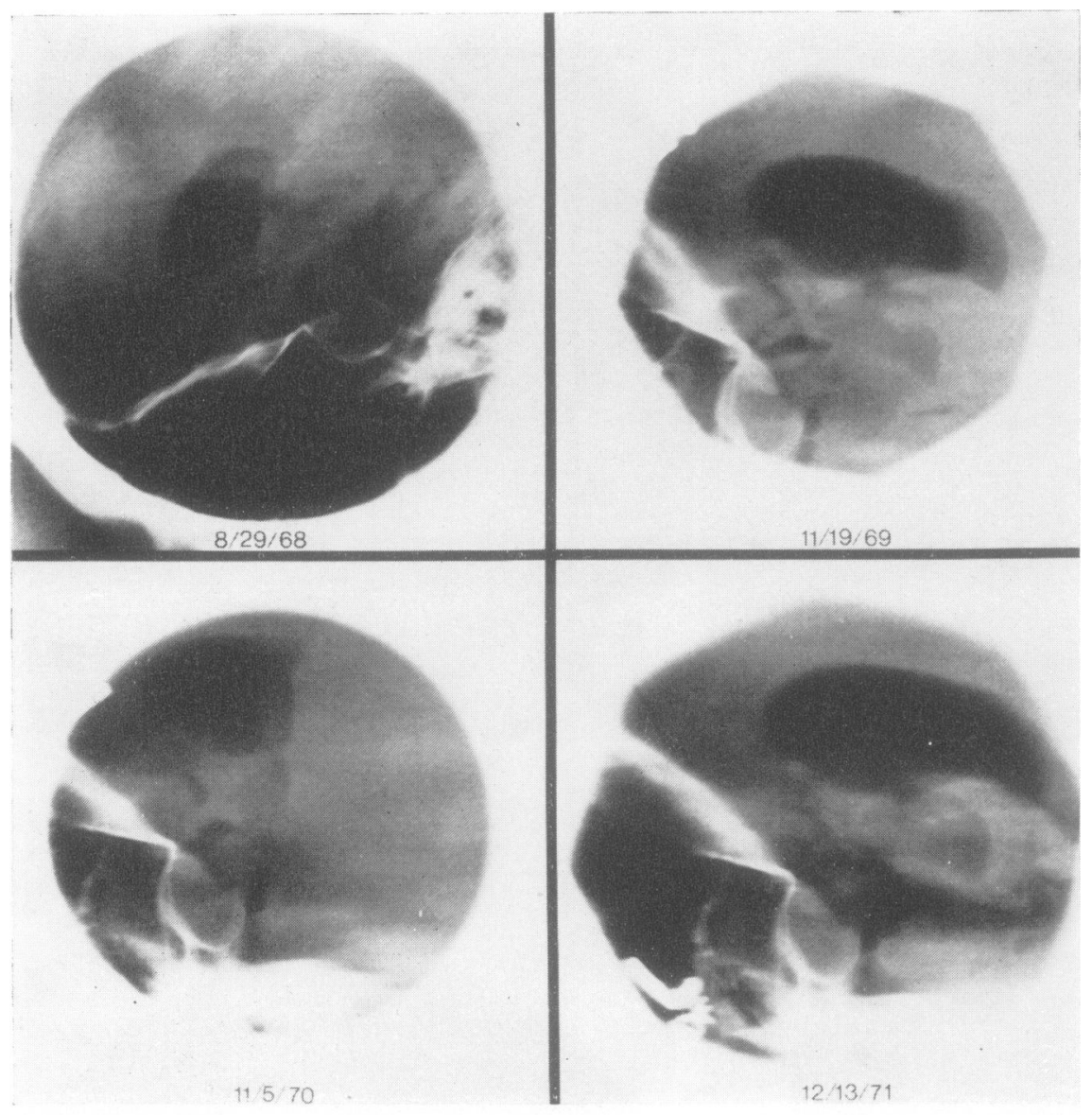

FIG. 4. Case 4 . (a, left) Serial pneumoencephalograms show suprasellar extension of intrasellar tumour after irradiation. The size does not appreciably change in the intervening three years. $(b$, bottom left) and (c, bottom right) Again suprasellar extension of intrasellar tumour is seen after transsphenoidal hypophysectomy and postoperative irradiation. Arrows indicate $\omega_{y}$ the suprasellar exte sion. The tumour appears to be slight

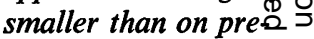
vious pneumoencephalography.

(a)

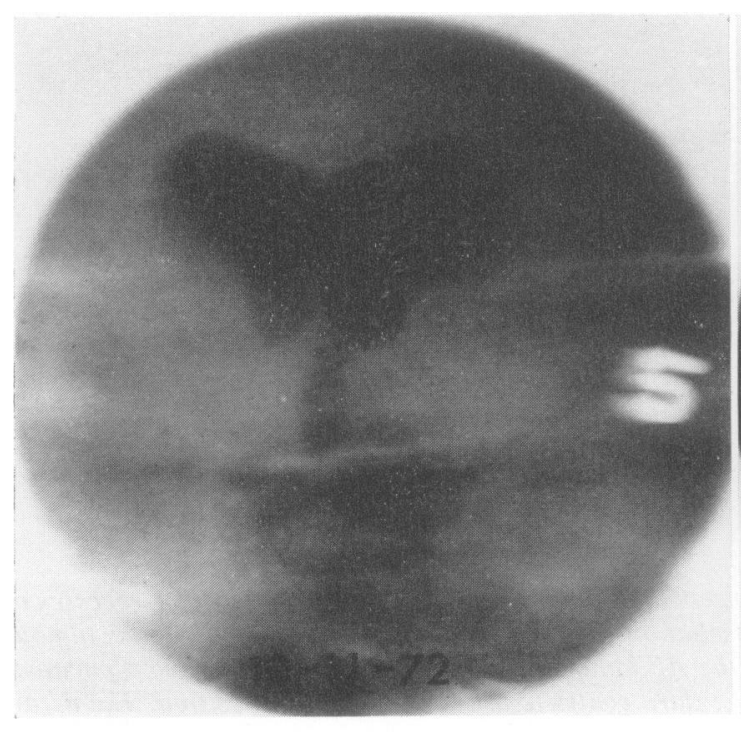

(b)

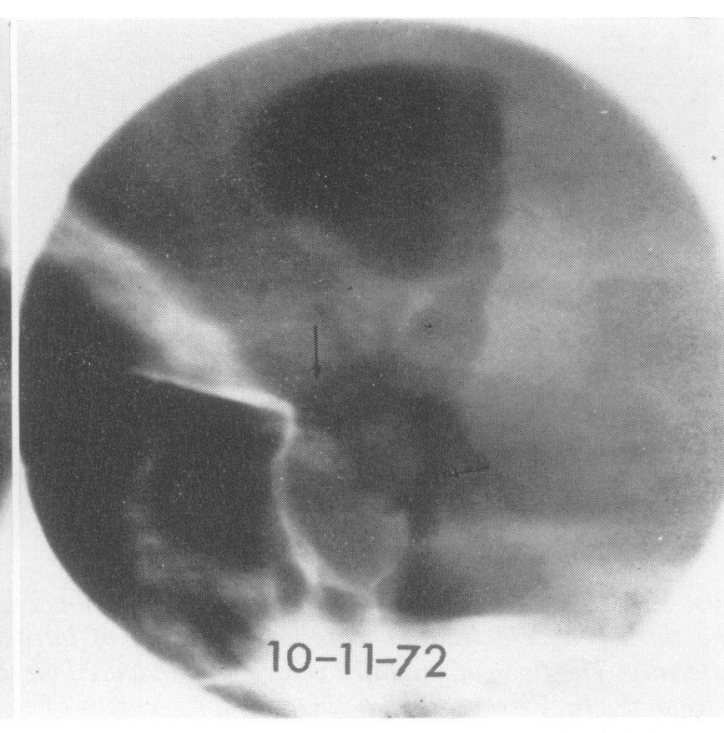

(c) 
6 November 1970 showed an intrasellar mass with suprasellar extension and deviation of the floor of the third ventricle from right to left. Endocrinological tests were normal. On 9 November 1970 the patient underwent a right frontal temporal craniotomy with subtotal excision of a chromophobe adenoma. All suprasellar extension of the mass was removed. The patient did well postoperatively and a partial ('limited') pneumoencephalogram was performed on 1 December 1970 to document removal of a suprasellar tumour, thus allowing focal irradiation to the sellar area. The follow-up 'limited' pneumoencephalogram demonstrated no superior extension of tumour. The third ventricle had returned to the midline (Fig. 1).

The 'limited' pneumoencephalogram in this case demonstrates what is expected to be seen after excision of nonsecretory tumour-that is, no tumour remaining in the suprasellar area. Focal irradiation can then be delivered to the sella turcica to eradicate any tumour remaining in the sella.

\section{CASE 2}

S.H., was a 68 year old male who entered with visual deterioration in the left eye over the year before admission. Seven years previously he had entered another hospital with symptoms of visual deterioration in the left eye with optic atrophy. Skull films demonstrated an enlarged sella turcica and carotid angiography demonstrated vascular displacement compatible with a pituitary tumour. A left frontal craniotomy was performed and a large chromophobe adenoma was removed. Surgical clips were left on the capsule of the tumour. The patient received postoperative irradiation (4000 $r$ tumour dose). During the year before the present admission vision in the left eye had deteriorated further. The patient retained light perception only. General physical examination was unremarkable. Neurological examination revealed light perception only in the left eye and a right temporal field cut. Carotid angiography demonstrated elevation of the first portion of the anterior cerebral arteries bilaterally. Pneumoencephalography revealed a large intrasellar mass with suprasellar extension. The clips which were previously placed on the capsule of the tumour at the patient's first operation were seen to lie just anterior to the dorsum sellae (Fig. 2). He successfully underwent trans-sphenoidal resection of recurrent pituitary tumour. Postoperative coned down films of the sella turcica showed no significant change in the position of the metallic clips after the trans-sphenoidal resection. Vision was improved.

This case demonstrates the hazards of relying on clips placed on the capsule of the tumour at surgery when considering tumour recurrence. In this patient there was significant tumour recurrence superiorly on the 'limited' pneumoencephalogram. However, from inspection of the surgically placed clips it would appear that there was little or no suprasellar extension.

\section{CASE 3}

L.B., was a 45 year old woman who entered with the complaint of headaches. She had undergone transfrontal hypophysectomy in 1970 for treatment of recurrent chromophobe adenoma. She also received irradiation in 1965 and 1970. She was readmitted with complaints of recurrent headaches for six months to rule out further recurrence of tumour. A partial pneumoencephalogram was performed which demonstrated no tumour recurrence and an 'empty sella' (Fig. 3). She was subsequently discharged without further therapy.

The value of the 'limited' pneumoencephalogram is exemplified by this case in which there were symptoms of recurrent tumour but no recurrence was demonstrated. The benignancy of a 'limited' pneumoencephalogram allows studies whenever symptoms occur or for routine followup at specified intervals.

\section{CASE 4}

E.B., a 37 year old female, was admitted for reevaluation of known chromophobe adenoma. The patient related a 12 year history of headaches. Four years before this admission examination at UCLA revealed an enlarged sella turcica. Pneumoencephalography revealed suprasellar extension of an intrasellar tumour. The patient received 5,000 $r$ tumour dose of radiotherapy. Her headaches persisted requiring reevaluation by pneumoencephalogram four times. During the last admission she was treated by transsphenoidal hypophysectomy as well as postoperative irradiation for recurrence. Since the last admission the patient complained of persistent right retro-orbital headaches with occasional vomiting after eating.

'Limited' pneumoencephalography showed minimal suprasellar extension of the tumour. Compared with her previous pneumoencephalograms it was 
felt that there was less tumour visible at this time (Fig. 4). She tolerated the procedure well and was discharged home without further therapy.

The 'limited' pneumoencephalogram has been a very useful tool in this particular case. Since many sellar tumours cannot be totally eradicated by surgery or irradiation, they must be controlled, thus requiring surgery or radiation therapy at various times. The 'limited' pneumoencephalogram was innocuously used in this patient to inform the neurosurgeon when further therapy would be necessary for control of her tumour. The exact location for surgery or radiotherapy was also demonstrated.

\section{DISCUSSION}

At UCLA Center for the Health Sciences patients suspected of having pituitary tumours have a full neuroradiological workup since it is important to obtain all possible information concerning size and growth characteristics of the tumour before surgery or radiotherapy. A full series of skull radiographs and coned down views and/or laminograms of the sella turcica in both the anteroposterior (AP) and lateral projections are obtained. Attention is focused on erosion or expansion of the sella turcica, suprasellar calcifications, and any apparent soft tissue mass extending into the sphenoid sinus.

Pneumoencephalography is performed with special attention to the sella turcica area. Filling of the chiasmatic and interpeduncular cisterns and anterior third ventricle must be adequate to define superior and posterior extension of the tumour. Laminography of the parasellar area in both the AP and lateral projections is obtained to define tumour extent more accurately. Views of the temporal horns are also important to evaluate any degree of lateral extension.

Carotid angiography is performed not only to define the vascular anatomy in the sella turcica region but also to exclude other lesions which may mimic the sellar changes seen with pituitary tumours such as aneurysm of the carotid artery or meningioma. Carotid arteriography also aids in defining the extent of a sellar lesion. Attention is paid to the distal internal carotid arteries (lateral extent), A1 portions of the anterior cerebral arteries (superior extent), and anterior choroidal arteries (posterior extent). If cryohypophysectomy is to be performed it is important to know the relationship of the internal carotid arteries to the sella turcica in the AP projection. If a definite diagnosis of sellar or parasellar lesion has been made previously, the carotid arteriogram may be limited to an AP internal injection only.

At present pneumoencephalography appears to be the best diagnostic procedure for determining recurrence of nonsecretory pituitary tumours. As these patients have been fully studied before surgery or radiotherapy, it is not necessary to subject them to repeat full pneumoencephalography. The 'limited' approach gives adequate information about tumour recurrence and is sufficiently benign to be performed on an outpatient basis at regular intervals. The 'limited' pneumoencephalogram gives information concerning the extent of tumour regrowth and continuing radiation effect after radiotherapy. In addition, the presence of an empty sella, whic might affect the optic chiasm and therefore cause additional eye signs, can be determined (Lee an $\$$ Adams, 1968). We have not as yet seen any cases of recurrent nonsecretory adenomas in the absence of clinical signs and symptoms except in the case of the fourth patient. Because we are $\vec{\theta}$ now routinely studying post-therapy pituitary $f$ tumour patients at specified intervals in the absence of signs and symptoms, it is felt that as our series grows we will experience such cases.

Apart from post-pneumoencephalography headaches, the morbidity-that is, meningitis or herniation-associated with pneumoencephalograms at UCLA has been extremely low. Morbidity, including headaches, is further reduced by careful technique, the use of small quantities of oxygen, and by close observation in the immediate post-pneumoencephalogram period. The authors realize the potential hazards of performing serial partial pneumoencephalograms on asymptomatic outpatients. However, it is felt that the possible gain to the patient outweighs the $ᄋ$ small risk of the serial examination. It is also felt that the careful observation of the patient in the immediate post-pneumoencephalogram period and the close contact between patient and physician after the patient has been discharged will prevent any major complication from occurring. 


\section{REFERENCES}

Lee, W. M., and Adams, J. E. (1968). The empty sella syndrome. Journal of Neurosurgery, 28, 351-356.

Lewtas, N. A. (1966). Symposium on pituitary tumours. 2. Radiology in diagnosis and management. Clinical Radiology, 17, 149-153.
Lindgren, M., Löfgren, F. O., and Lundberg, N. (1957). Tantalum powder as an indicator of the brain tumour region for postoperative radiotherapy and the diagnosis of recurrence. Acta Radiologica, 48, 17-25.

Wilson, C. B., and Norrell, H. A. (1968). Radiographic marker for the early detection of recurrent pituitary tumors. Acta Neurochirurgica, 18, 293-296. 